\title{
CONSTRUÇÃO DE UM SUPORTE AJUSTÁVEL LAB-MADE IMPRESSO EM 3D PARA MEDIÇÃO DE ÂNGULO DE CONTATO
}

\author{
Vinicius A. O. P. da Silva ${ }^{a}$, Vinicius A. P. Tartare ${ }^{a}$ Cristiane Kalinke ${ }^{\mathrm{b}}$, Paulo Roberto de Oliveira ${ }^{\mathrm{a}}$, Daniel Cardoso de Souza, \\ Juliano A. Bonacin ${ }^{\mathrm{b}, *}$ e Bruno C. Janegitz ${ }^{\mathrm{a}, *,(\mathbb{D}}$ \\ aDepartamento de Ciências da Natureza, Matemática e Educação, Universidade Federal de São Carlos, 13600-970 Araras - SP, Brasil \\ 'Instituto de Química, Universidade Estadual de Campinas, 13083-970 Campinas - SP, Brasil
}

Recebido em 21/04/2020; aceito em 08/07/2020; publicado na web em 31/08/2020

\begin{abstract}
LAB-MADE 3D-PRINTED CONTACT ANGLE MEASUREMENT ADJUSTABLE HOLDER. Advances in the use of 3D printing technology have led to the development of new low-cost devices. This technology features high versatility, considering the easy modulation of the materials depending on its objectives and applications. In this sense, we have developed a laboratory-built contact angle measuring device (lab-made). The device was composed by the assembly of 26 3D-printed pieces of different sizes and design printed using acrylonitrile-butadiene-styrene polymer. The device was designed in three main parts. The first part was related to the analysis, with the use of platforms for the sample surfaces positioning. The second part was made to support a micropipette, prepared to add the drop for analysis; and the third for the support of a smartphone, utilized to obtain the images. The device was applied for the measurement of contact angle on different surfaces, demonstrating its versatility and precision of the results. The obtained values are in accordance with others found in the literature. The developed device presented a low-cost (US\$25.34 per device), easy assembly and operation.
\end{abstract}

Keywords: 3D printing; ABS polymer; lab-made; 3D device; contact angle.

\section{INTRODUÇÃO}

Nos últimos anos, a tecnologia de impressão em três dimensões (3D), também chamada de manufatura aditiva, tem emergido como uma ferramenta revolucionária e versátil para a produção tanto de peças e objetos simples, como dispositivos e equipamentos sofisticados. ${ }^{1,2}$ A grande vantagem do uso dessa ferramenta é a possibilidade de projetar peças de diferentes formatos e dimensões com o uso de softwares de design de objetos 3D. ${ }^{3}$ Outro ponto que fortalece o uso dessa tecnologia é o baixo custo e o tempo de impressão gasto. ${ }^{4,5}$ Devido a estas características, a impressão 3D vem sendo utilizada em diferentes aplicações, tais como na medicina, ${ }^{6,7}$ mecatrônica, ${ }^{8}$ robótica, ${ }^{9}$ educação, ${ }^{10}$ brinquedos e jogos, ${ }^{11}$ agricultura ${ }^{12}$ e química. ${ }^{13-15}$

Dentre algumas das técnicas para se imprimir objetos ou dispositivos em 3D, a técnica de modelagem de deposição por fusão (FDM, fused deposition modeling) tem ganhado espaço neste campo. ${ }^{16,17}$ Essa técnica consiste no uso de um filamento polimérico termoplástico que quando aquecido pode ser extrusado e depositado, camada por camada, sobre uma plataforma de impressão. ${ }^{13,18} \mathrm{O}$ design ou desenho do objeto normalmente é projetado em softwares do tipo CAD (Computer-Aided Design), apropriados para desenhos em $3 \mathrm{D} .{ }^{19} \mathrm{O}$ projeto é então exportado em um formato que o software da impressora 3D reconheça. Nesta etapa é possível alterar configurações da impressão, como velocidade da impressão, espessura das camadas impressas, ajuste de temperatura do bico de extrusão e da plataforma de impressão, entre outras funções, que otimizam o processo de impressão. $^{20,21}$

A versatilidade da tecnologia de impressão em 3D vem atraindo cada vez mais a atenção da comunidade acadêmica, sendo uma estratégia comumente utilizada para a obtenção de objetos e dispositivos de uso laboratorial. Dentre estas aplicações, a impressão 3D contribuiu para o desenvolvimento de equipamentos

\footnotetext{
*e-mail: jbonacin@unicamp.br; brunocj@ufscar.br
}

de uso laboratorial, ${ }^{22}$ sensores eletroquímicos ${ }^{23-25}$ e eletrônicos, ${ }^{26}$ capacitores,${ }^{27}$ dispositivos microfluídicos, ${ }^{28,29}$ células eletroquímicas, ${ }^{30}$ células espectroeletroquímicas, ${ }^{3}$ microscópios eletrônicos, ${ }^{31}$ entre outros.

Seguindo esse raciocínio, dos Santos e colaboradores ${ }^{3}$ desenvolveram uma célula espectroeletroquímica impressa em 3D utilizando filamento de acrilonitrila-butadieno-estireno (ABS, acrylonitrile-butadiene-styrene) e filamento condutor de ácido polilático (PLA, polylactic acid) com grafeno. O dispositivo foi facilmente construído e teve sucesso em medidas de Raman in situ para a determinação da alteração estrutural de materiais em reações eletroquímicas, tal como para a análise do mecanismo dos processos de disposição e adsorção de filmes na superfície de eletrodos. Em outro trabalho, Schneidereit e colaboradores ${ }^{31}$ projetaram um microscópio eletrônico automatizado. O dispositivo foi impresso em 3D utilizando aproximadamente $250 \mathrm{~g}$ de filamento ABS, e com tempo total de impressão de 23 horas. Os autores relataram que o microscópio apresentou fácil construção, portabilidade, versatilidade para aplicação em diversas áreas, e ainda mostrou eficiência comparável a outros instrumentos comerciais.

Assim, fica evidente que a tecnologia 3D é eficaz para a construção de dispositivos de maneira rápida e com baixo custo, permitindo explorar a sua versatilidade e capacidade de modulação de designs. Neste sentido, nós desenvolvemos pela primeira vez um dispositivo 3D, de forma simples e com baixo custo, para análises de ângulo de contato, com a finalidade de determinar a molhabilidade de superfícies variadas. Ângulo de contato é conhecido como o ângulo formado quando uma gota de um líquido é depositada sobre uma superfície sólida e plana, o qual é delimitado entre a superfície e uma reta tangente à superfície da gota. ${ }^{32} \mathrm{O}$ ângulo de contato é um indicador do caráter hidrofóbico ou hidrofílico de uma superfície, dependendo da interação da superfície em contato com o líquido. ${ }^{33}$ Neste sentido, o ângulo de contato de $90^{\circ}$ é o ponto de divisão teórico para a definição de hidrofobicidade ou hidrofilicidade da superfície para um determinado líquido. ${ }^{32}$ Assim, o ângulo de 
contato zero caracteriza que o líquido "molhou" o material e há a tendência de um espalhamento do mesmo na superfície. Já a molhabilidade parcial é caracterizada por um ângulo intermediário, entre $0^{\circ}$ e $180^{\circ}\left(0^{\circ}<\theta<180^{\circ}\right) .^{34}$ Porém, alguns fatores, tais como a possível contaminação do substrato, irregularidades na superfície (rugosidade, tamanho e formato das partículas do sólido), alterações ou modificações superficiais podem influenciar no comportamento de molhabilidade superficial, consequentemente, afetando a determinação precisa do ângulo de contato. ${ }^{35}$ Assim, as medidas de ângulo de contato, normalmente, são realizadas com auxílio de equipamentos específicos, tal como o goniômetro. Contudo, estes equipamentos necessitam de um técnico treinado para a operação e podem apresentar elevado valor agregado. Estas desvantagens podem ser contornadas com o desenvolvimento de dispositivos do tipo lab-made, impressos em 3D. Neste trabalho, há proposição de um dispositivo para a medição de ângulo de contato visando à autonomia laboratorial, satisfazendo algumas características, como versatilidade, mobilidade, precisão, facilidade de montagem e operação e baixo custo. O objetivo deste trabalho foi possibilitar a impressão 3D de um suporte para smartphone e micropipeta que permita a análise de molhabilidade de superfícies por ângulo de contato, como uma alternativa para equipamentos comerciais de alto custo.

\section{PARTE EXPERIMENTAL}

\section{Materiais}

O dispositivo foi impresso em uma impressora 3D utilizando filamento polimérico ABS obtido pela UP3D (São Paulo, Brasil). Cola em bastão Scotch (3M, Brasil) foi utilizada para a aderência das peças à plataforma de impressão. Água ultrapura $(>18 \mathrm{M} \Omega \mathrm{cm})$ obtida por sistema Milli-Q Plus (Merck Millipore, EUA) foi utilizada para a formação da gota utilizada para a medição do ângulo de contato.

Peças impressas em 3D, obtidas com filamentos de polímeros comerciais ABS e PLA (Sethi 3D, Brasil) e folha de papel tipo office com gramatura de $75 \mathrm{~g} \mathrm{~m}^{-2}$ (Tilibra, Brasil) foram utilizados para as análises de ângulo de contato. Superfícies eletródicas foram também avaliadas, sendo estas: eletrodo de carbono vítreo (GCE, do inglês glassy carbon electrode), eletrodos impressos em substrato de papel impermeável (WPE, do inglês waterproof paper electrode) e de cloreto de polivinila (PVCE, do inglês polyvinyl chloride electrode).

\section{Design do dispositivo 3D para medição de ângulo de contato}

A elaboração do design do protótipo foi feita com auxílio dos softwares Inventor Professional 2018 e AutoCAD 2019 (Autodesk, EUA), inicialmente desenhado com ferramentas de linhas e curvas 2D. Em seguida, os desenhos foram transformados em 3D e salvos em formato Surface Tessellation Language. O desenho do dispositivo foi convertido em um modelo de camadas utilizando o software Simplify 3D, acoplado à impressora 3D. A montagem das peças do dispositivo pode ser visualizada no Video V1 (Material Suplementar). O design final do dispositivo foi composto por três partes principais: uma plataforma para a adição da gota e medição do ângulo de contato das superfícies (a); um suporte para o acoplamento de uma micropipeta utilizada para a adição precisa da gota (b) e um suporte para smartphone, utilizado para a obtenção das imagens de ângulo de contato (c) (Figura 1). O dispositivo apresentou dimensões gerais de $385 \mathrm{~mm}$ de altura, $260 \mathrm{~mm}$ de profundidade de $135 \mathrm{~mm}$ de largura.

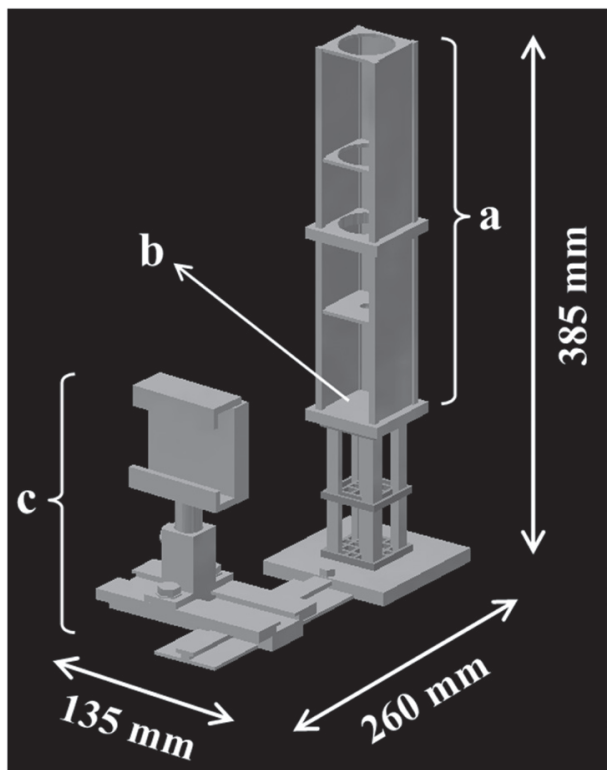

Figura 1. Design do dispositivo para medida de ângulo de contato projetado para a impressão $3 D$, obtido com o software Simplify $3 D^{\circledR}$

\section{Impressão das peças e montagem do dispositivo}

A impressão do dispositivo foi realizada utilizando uma impressora 3D SETHI 3D S3 (Sethi3D, Brasil). A técnica de FDM foi utilizada para a impressão do dispositivo devido as suas vantagens como elevada acessibilidade, relativo baixo custo e fácil manuseio. ${ }^{16,20,21}$ Esta técnica vem sendo amplamente empregada para impressão 3D dentre as técnicas que requerem o uso de filamentos poliméricos termoplásticos, tal como o ABS utilizado neste trabalho. Antes da impressão, uma fina camada de cola em bastão foi aplicada sobre a plataforma de impressão. Esse procedimento foi realizado antes de cada impressão, e contribuiu para a melhor aderência das peças à plataforma, impedindo a sua movimentação durante o processo de impressão.

O dispositivo foi composto por 26 peças, em que a maioria dos parâmetros de impressão foi mantida nos padrões predefinidos pelo software Simplify 3D para o filamento de ABS. Os parâmetros diâmetro do bico de extrusão, temperatura do bico de extrusão, temperatura da plataforma de impressão e velocidade de impressão foram alterados para garantir a melhor qualidade das peças. Devido à elevada temperatura do bico de extrusão, o cooler de resfriamento do bico foi mantido desligado durante todo o processo de impressão com o intuito de evitar possíveis falhas e rachaduras nas peças, causadas por choque térmico no material. Os parâmetros avaliados, tal como suas condições de compromisso podem ser observados na Tabela 1.

\section{Medidas de ângulo de contato e processamento das imagens}

A análise de ângulo de contato foi realizada em diferentes superfícies, visando a avaliação de seu caráter hidrofóbico ou hidrofílico. As seguintes superfícies foram analisadas: ABS, PLA, papel tipo office, eletrodo de carbono vítreo (GCE), eletrodo impresso em papel impermeável (WPE) e eletrodo impresso em PVC (PVCE). Para o registro das imagens o equipamento foi projetado para acoplar um smartphone para fotografar a gota na superfície analisada. O tratamento das imagens foi feito com auxílio do software CorelDRAW Graphics Suite 2019 (Corel Corporation, Canadá). É importante ressaltar que a utilização de outros softwares para o tratamento das imagens também seria possível, principalmente utilizando programas com licença gratuita, tal como o software de domínio público ImageJ. 
Tabela 1. Principais parâmetros de impressão 3D utilizados para a confecção do dispositivo

\begin{tabular}{lc}
\hline Parâmetro & Condição de compromisso \\
\hline Diâmetro do bico de extrusão & $0,40 \mathrm{~mm}$ \\
Altura da primeira camada & $200 \%$ \\
Largura da primeira camada & $100 \%$ \\
Velocidade da primeira camada & $50 \%$ \\
Padrão do preenchimento & Retilíneo \\
Preenchimento interno & $30-50 \%$ \\
Temperatura do bico de extrusão & $225^{\circ} \mathrm{C}$ \\
Temperatura da plataforma de impressão & $90{ }^{\circ} \mathrm{C}$ \\
Velocidade de impressão & $800-3600 \mathrm{~mm} \mathrm{~min}^{-1}$ \\
Velocidade de movimentação dos eixos x e y & $5000 \mathrm{~mm} \mathrm{~min}^{-1}$ \\
Velocidade de movimentação do eixo z & $1002 \mathrm{~mm} \mathrm{~min}^{-1}$ \\
\hline
\end{tabular}

\section{RESULTADOS E DISCUSSÃO}

\section{Impressão e montagem do dispositivo 3D}

A impressão do dispositivo 3D para medição de ângulo de contato foi realizada utilizando parâmetros de impressão padrões para impressão de objetos em polímero ABS, avaliados de acordo com o apresentado na Tabela 1. Os seguintes parâmetros de impressão foram fixados para a obtenção do dispositivo proposto: diâmetro do bico de extrusão de $0,40 \mathrm{~mm}$, temperatura do bico de extrusão de $225^{\circ} \mathrm{C}$, temperaturas da plataforma de impressão de $90{ }^{\circ} \mathrm{C}$ e velocidade de impressão de 800 a $3600 \mathrm{~mm} \mathrm{~min}^{-1}$.

Para a obtenção deste dispositivo foi utilizado um bico de extrusão com diâmetro de $0,40 \mathrm{~mm}$, sendo que este pode ser variável de acordo com o diâmetro do filamento utilizado para a impressão.
A temperatura de trabalho é um fator predominante, como discutido também em outros trabalhos, ${ }^{36,37}$ e que foi considerada para manter a qualidade das peças impressas. Para isso, a temperatura ambiente foi controlada em $25^{\circ} \mathrm{C}$, e as temperaturas do bico de extrusão e da plataforma de impressão foram fixadas em $225^{\circ} \mathrm{C}$ e $90{ }^{\circ} \mathrm{C}$, respectivamente, para todas as impressões. Além disso, após as impressões as peças permaneceram na plataforma de impressão até o resfriamento, sem intervenção externa. A impressora permaneceu fechada (a mesma possui uma porta frontal) durante todo o processo de impressão e resfriamento das peças. A velocidade de impressão foi alterada de acordo com a peça a ser impressa. Neste caso, peças que demandavam maiores detalhes e encaixes mais precisos foram obtidas em velocidade menor (800-2500 $\left.\mathrm{mm} \mathrm{min}^{-1}\right)$, comparadas às peças que não demandavam maior precisão (3000-3600 $\left.\mathrm{mm} \mathrm{min}^{-1}\right)$. A necessidade de modulação da velocidade de impressão, em virtude da maior ou menor precisão requerida das peças impressas, também é algo já explorado na literatura. ${ }^{38} \mathrm{~A}$ Tabela 2 detalha as peças impressas com as respectivas dimensões e velocidades de impressão. A Figura 2 ilustra todas estas peças enumeradas conforme a relação da Tabela 2. No Material Suplementar - S1 é possível obter maiores informações para a impressão das peças do dispositivo. Os arquivos para a impressão das peças também estão disponíveis.

O dispositivo foi montado seguindo o passo-a-passo apresentado na Figura 3. Inicialmente, o trilho do eixo y foi anexado à base de fixação (1), para dar maior sustentação ao dispositivo durante a montagem. Em seguida, os pilares central (2) e externos (3) foram fixados na base. Duas cintas de apoio foram adicionadas internamente e externamente aos pilares, visando a melhor fixação dos mesmos (4). Então, a base de deposição da solução foi acomodada sobre os pilares (5). O trilho do eixo $\mathrm{x}$ foi encaixado ao trilho do eixo y (6). A seguir, o suporte para o smartphone foi montado (7). Para isso, o suporte superior foi encaixado ao suporte superior, e o cilindro para ajuste do eixo z foi fixado ao suporte inferior. Em seguida, o cilindro foi encaixado à base para ajuste do eixo z. Um parafuso de $7,0 \mathrm{~mm}$ foi utilizado para fixação do cilindro à base e para o ajuste

Tabela 2. Detalhamento das peças impressas para a confecção do dispositivo, contendo dimensões $(\mathrm{A} \times \mathrm{L} \times \mathrm{P}$ ou $\mathrm{A} \times \mathrm{D})$ e respectivas velocidades de impressão

\begin{tabular}{|c|c|c|c|}
\hline $\mathrm{N}^{\mathrm{o}}$ & Peça & Dimensão (mm) & Velocidade de impressão $\left(\mathrm{mm} \mathrm{min}^{-1}\right)$ \\
\hline 1 & Base de fixação & $10,0 \times 100 \times 100$ & 3600 \\
\hline 2 & Pilar de sustentação central & $109 \times 9,95 \times 9,95$ & 2500 \\
\hline 3 & Pilares de sustentação externos (4) & $109 \times 9,95 \times 9,95$ & 2500 \\
\hline 4 & Base de deposição da solução & $8,00 \times 59,5 \times 59,5$ & 3000 \\
\hline \multirow{2}{*}{5} & Cinta de apoio externo & $5,00 \times 44,0 \times 44,0$ & 3600 \\
\hline & Cinta de apoio interno & $5,00 \times 28,97 \times 28,97$ & 3600 \\
\hline 6 & Trilho do eixo y & $8,70 \times 50,0 \times 170$ & 3500 \\
\hline 7 & Trilho do eixo $\mathrm{x}$ & $19,1 \times 135 \times 66,0$ & 3000 \\
\hline 8 & Base para ajuste do eixo z & $52,6 \times 66,0 \times 26,0$ & 2000 \\
\hline \multirow{2}{*}{9} & Cilindro para ajuste do eixo $\mathrm{z}$ & $63,5 \times 19,8$ & 2000 \\
\hline & Suporte para smartphone inferior & $70,0 \times 75,0 \times 35,0$ & 3600 \\
\hline 10 & Suporte para smartphone superior & $65,0 \times 70,0 \times 29,7$ & 3600 \\
\hline 11 & Suporte inferior micropipeta & $137 \times 49,5 \times 49,5$ & 3600 \\
\hline 12 & Suporte superior micropipeta & $130 \times 49,5 \times 49,5$ & 2500 \\
\hline 13 & Cinta de encaixe micropipeta & $5,00 \times 44,6 \times 44,6$ & 2500 \\
\hline 14 & Parafuso $10 \mathrm{~mm}(2)$ & $15,0 \times 12,7$ & 800 \\
\hline 15 & Parafuso 7,0 mm (2) & $15,0 \times 16,2$ & 800 \\
\hline 16 & Pino de travamento do trilho y & $14,5 \times 11,6$ & 800 \\
\hline
\end{tabular}

A: altura; L: largura; P: profundidade; D: diâmetro; $\mathrm{N}^{\circ}$ : número da peça. 


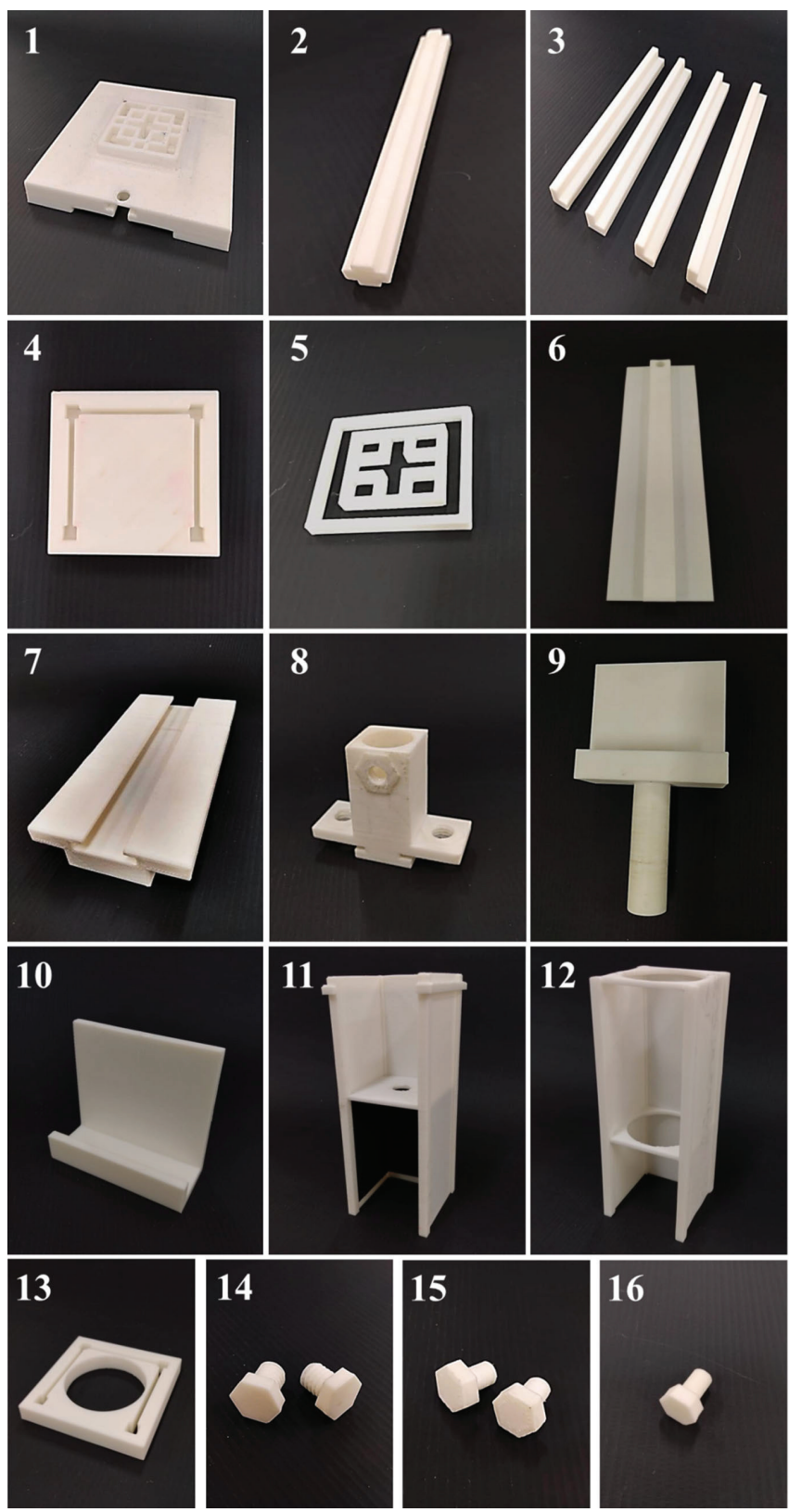

Figura 2. Peças impressas em 3D para a confecção do dispositivo para medição de ângulo de contato

de altura do eixo z. Um segundo parafuso foi utilizado para a fixação dos suportes para smartphone (parte posterior), conforme mostrado pela ampliação. O suporte para o smartphone foi então encaixado ao trilho do eixo y, utilizando dois parafusos de $10 \mathrm{~mm}$ (8). Então, foi realizada a montagem do suporte para a micropipeta (9). Os suportes inferior e superior foram encaixados com o auxílio de uma cinta para fixá-los entre si, conforme mostrado na imagem inserida. Um papel autoadesivo preto foi colado na parte interna no suporte inferior para a melhor obtenção das imagens. Por fim, o suporte para micropipeta foi encaixado à base de deposição. A Figura 4 mostra a visão geral do dispositivo pronto para uso, após a montagem de todas as peças impressas em 3D e contendo um smartphone e micropipeta acoplados.

$\mathrm{O}$ dispositivo 3D foi composto por três partes, sendo a primeira destas a plataforma de medida, a segunda o suporte para micropipeta e a terceira o suporte para smartphone. A seguir cada parte será descrita separadamente. A plataforma de medida foi composta pela base para a adição da gota nas superfícies a serem analisadas. Esta
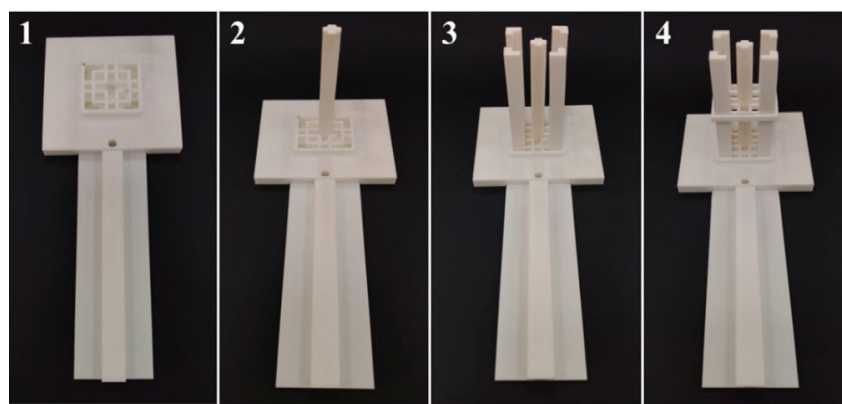
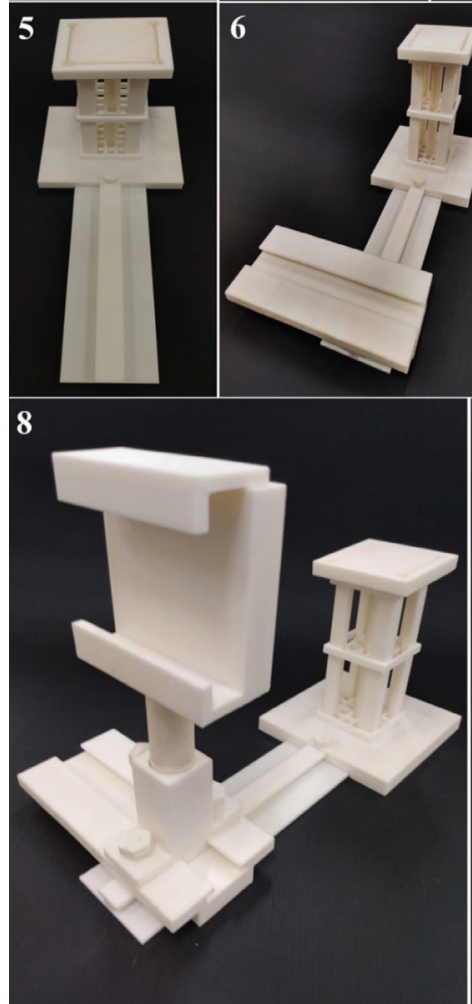
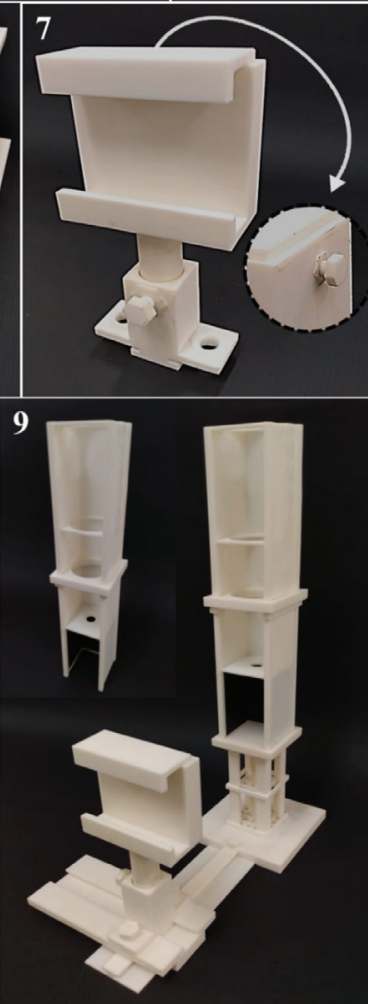

Figura 3. Passo-a-passo da montagem do dispositivo 3D para medição de ângulo de contato

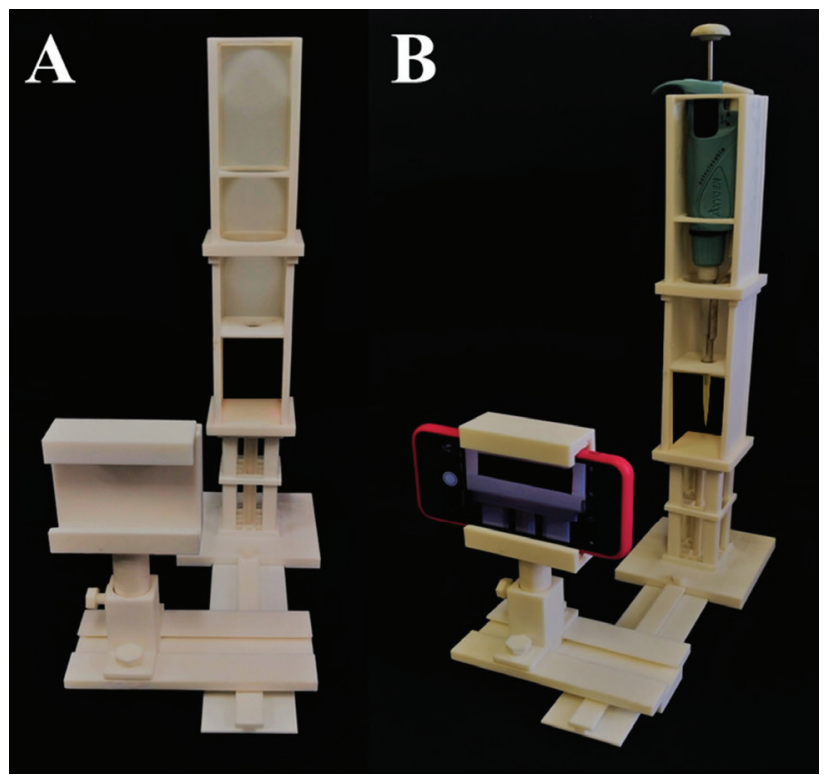

Figura 4. Dispositivo para medição de ângulo de contato impresso em $3 D$ utilizando filamento ABS: visão frontal (A) e lateral, contendo um smartphone e micropipeta acoplados $(B)$ 
base apresentou dimensões de 59,5 mm de largura e profundidade, o que facilitou o posicionamento de diferentes materiais para a análise superficial. A base foi elevada e sustentada por cinco pilares de $109 \mathrm{~mm}$ de altura, permitindo o seu apoio sobre a base principal. Por fim, as cintas de apoio foram confeccionadas e posicionadas entre as duas bases, visando a melhor sustentação e estabilidade da plataforma (Figura 5A). Uma segunda plataforma de medição foi também confeccionada nas mesmas dimensões da primeira e contendo um orifício de 7,0 mm de diâmetro, possibilitando o acoplamento de eletrodos e outras superfícies cilíndricas (Figura 5B).
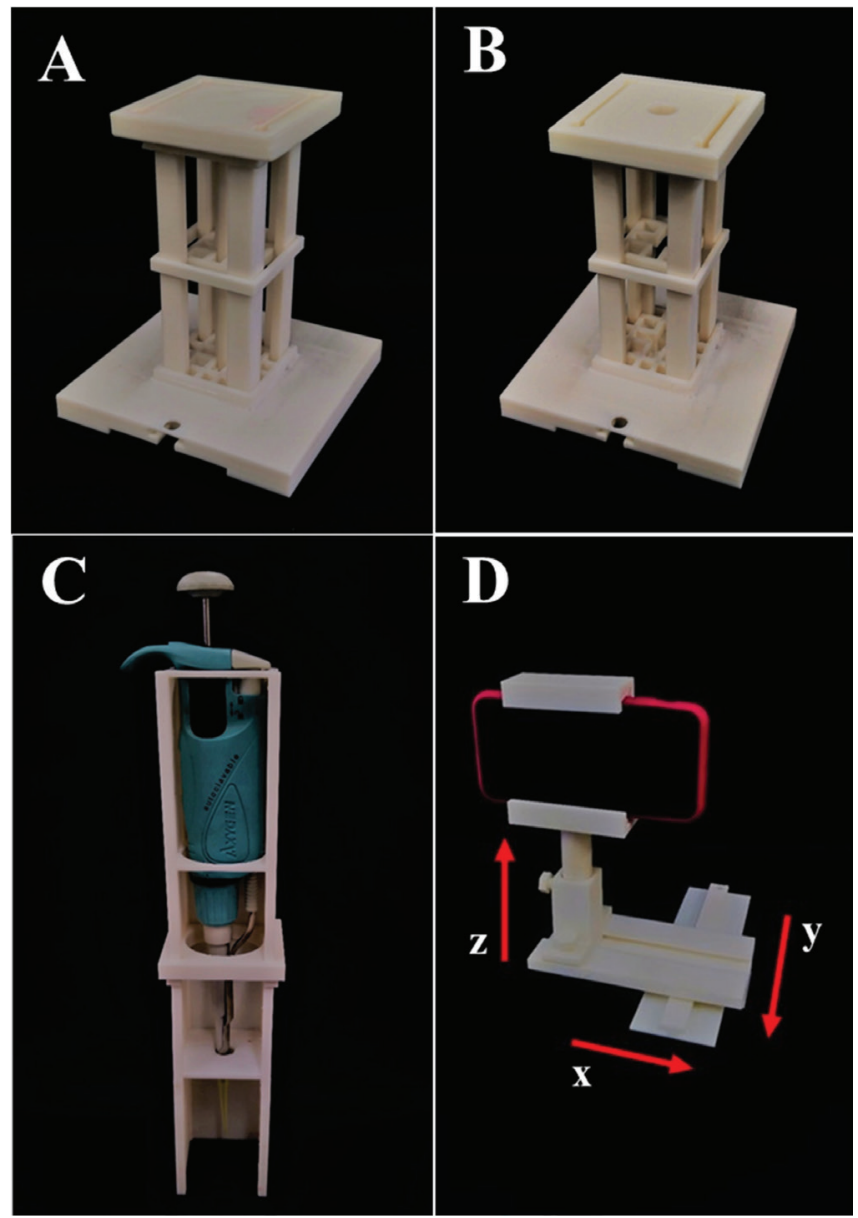

Figura 5. Dispositivo $3 D$ para medição de ângulo de contato: bases de deposição da solução (gota) para superfícies planas (A) e cilíndricas (B), suportes para micropipeta $(C)$ e smartphone $(D)$

Para tornar a adição do líquido analisado (gota) à superfície de maneira mais precisa e simplificada, uma peça específica para sustentar a micropipeta foi também impressa (Figura 5C). A utilização de um suporte fixo para o sistema de injeção do líquido já foi abordada em outros trabalhos, tal como o apresentado por Chen e colaboradores, ${ }^{39}$ em que a presença deste suporte permitiu um melhor controle e repetibilidade do tamanho da gota formada. O presente dispositivo apresentou dimensões de $267 \mathrm{~mm}$ de altura e 49,5 mm de largura e profundidade, o que possibilitou o acoplamento de micropipetas com volumes distintos. Testes foram realizados utilizando micropipetas das marcas Axygen, Dragonlab e Kasvi, com variação de volumes de 0,1-10, 10-100 e 20-200 $\mu \mathrm{L}$. Para este estudo uma micropipeta com volume de $10-100 \mu \mathrm{L}$ foi utilizada para a adição de uma alíquota de $10 \mu \mathrm{L}$ de água deionizada às superfícies analisadas.

A aquisição das imagens para o cálculo do ângulo obtido entre a superfície e a gota depositada foi realizada com o auxílio da câmera de um smartphone. $\mathrm{O}$ aparelho foi fixado em um suporte com ajuste de posição nos eixos x, y e z (largura, profundidade e altura) (Figura 5D). A utilização de smartphone em medidas de ângulo de contato já foi relatada na literatura. ${ }^{39}$ No entanto, o arranjo das peças impressas para os dispositivo proposto possibilitou um melhor alinhamento no posicionamento do smartphone em relação ao nível da base onde o líquido foi depositado, levando a maior precisão do cálculo do ângulo formado entre a gota e a superfície analisada. Além disso, a possibilidade do ajuste em três diferentes eixos permitiu uma melhor mobilidade do smartphone, em comparação a outros suportes estáticos reportados pela literatura. ${ }^{39}$

O dispositivo proposto foi desenvolvido para suprir a necessidade de pesquisadores em medições de ângulo de contato. Neste sentido, o dispositivo pôde ser facilmente projetado e construído com peças impressas em 3D, e que apresentaram relativo baixo custo em comparação a um equipamento comercial, com custo médio de US\$ 7.434,94. A Tabela 1S apresenta a relação das peças impressas para a confecção do dispositivo, contendo o tempo de impressão, material gasto e a estimativa de custo unitário e total. O dispositivo para medição de ângulo de contato apresentou um tempo total para impressão das 26 peças de aproximadamente 66 horas, considerando que as peças foram obtidas em alta qualidade de impressão (menor velocidade de impressão), resultando no custo aproximado de US\$25,34 por dispositivo.

Considerando ser um dispositivo lab-made, com etapas de montagem artesanais e fácil produção, a comparação com equipamentos comerciais produzidos industrialmente fica limitada ao custo final de produção e facilidade de operação. Enquanto equipamentos industriais necessitam de um profissional capacitado para operá-lo, o dispositivo proposto possui uma operação simples, rápida e intuitiva. Não há necessidade de um profissional capacitado para ser operado, sendo suficiente apenas seguir as orientações prévias quanto à montagem do dispositivo e o ajuste da angulatura do sensor de captura da imagem. É importante ressaltar que o custo final estimado do dispositivo 3D lab-made não considerou custos de operadores, tempo e energia gastos. Nós partimos do pressuposto de que o dispositivo poderá ser impresso para uso próprio em laboratório de ensino, pesquisa e extensão, sem produção em larga escala ou comercialização. Portanto o custo de produção total referente ao dispositivo proposto para fins exclusivos de medição de ângulo de contato é de aproximadamente $0,26 \%$ do valor da compra de um goniômetro comercial. O dispositivo proposto se trata de uma alternativa para a análise de molhabilidade de superfícies por ângulo de contato com baixo custo, não tendo como fim a substituição do equipamento comercial em sua ampla funcionalidade com custo de alguns milhares de dólares.

\section{Aplicação do dispositivo 3D: medidas de ângulo de contato}

Para as medidas de ângulo de contato, os materiais foram centralizados na plataforma de medição do dispositivo. Em seguida, com o auxílio de uma micropipeta, uma alíquota de $10 \mu \mathrm{L}$ de água deionizada foi adicionada à superfície dos materiais, formando uma gota. A gota permaneceu na superfície das amostras por 5 segundos antes da captura das imagens utilizando um smartphone. $\mathrm{O}$ ângulo de contato foi obtido entre a gota e a superfície. Para isso, uma linha foi traçada sobre o mesmo plano horizontal onde a gota foi depositada, e outra linha tangente à superfície da gota, formando a abertura do ângulo, conforme exemplificado na Figura 6A. Em posse do valor do ângulo de contato formado $(\theta)$ e da tensão superficial do líquido em relação ao gás $\left(\gamma_{\mathrm{LG}}\right)$, foi possível calcular o trabalho de adesão da gota à superfície $\left(\tau_{\mathrm{LS}}\right)$ utilizando a Equação de Young (Equação $\left.(1)\right){ }^{34}$ Assim, a molhabilidade de uma superfície pode ser classificada de 

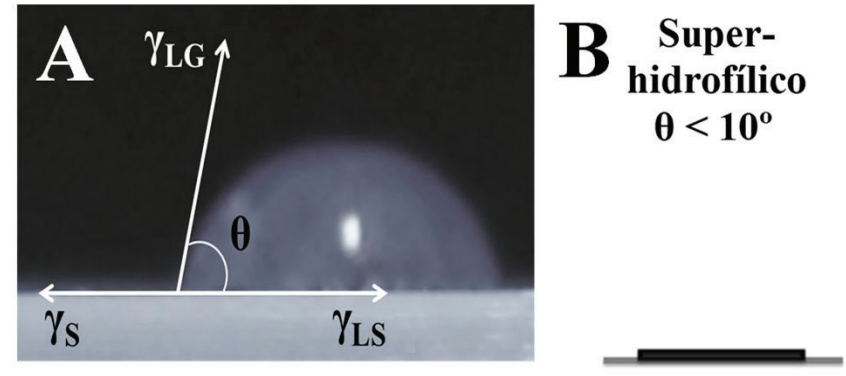
Hidrofílico $\boldsymbol{\theta}<\mathbf{9 0}^{\circ}$ Hidrofóbico $\mathbf{9 0}^{\circ}<\boldsymbol{\theta}<150^{\circ}$
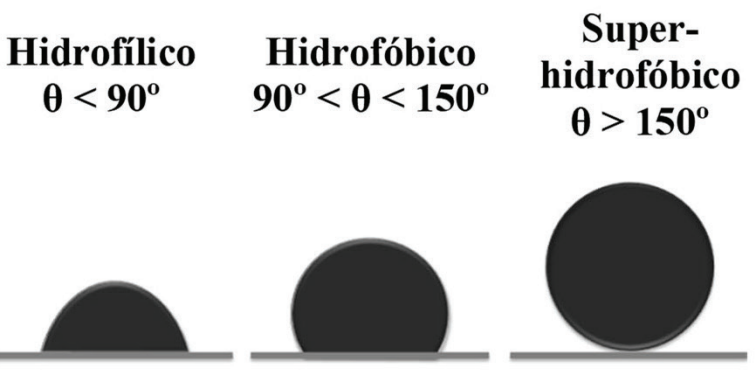

Figura 6. Obtenção do ângulo de contato $(\theta)\left(\gamma_{S}\right.$ : energia de superfície do sólido; $\gamma_{L G}$ : tensão superficial do equilíbrio líquido-gasoso; $\gamma_{L S}$ : energia da interface líquido-sólido) (A). Classificação do ângulo de contato obtido para diferentes comportamentos de fluidos com uma superfície (B)

acordo com o ângulo de contato. A Figura 6B apresenta a classificação das características superficiais para diferentes comportamentos de fluidos, relacionado com o ângulo de contato formado. Neste caso, superfícies com $\theta<10^{\circ}$ apresentam caráter super-hidrofílico, $\theta<90^{\circ}$ hidrofílico, $90^{\circ}<\theta<150^{\circ}$ hidrofóbico e $\theta>150^{\circ}$ super-hidrofóbico. ${ }^{40}$

$$
\tau_{\mathrm{LS}}=\gamma_{\mathrm{LG}}(1+\cos \theta)
$$

A Tabela 3 apresenta os resultados obtidos para as medidas de ângulo de contato $(\theta)$ em diferentes materiais: ABS, PLA, papel tipo office, eletrodos GCE, WP, PVC e PET. O ABS é um copolímero sintético obtido por reações de adição dos monômeros acrilonitrila, butadieno e estireno. Este termoplástico é amplamente utilizado na indústria e na impressão de objetos em $3 \mathrm{D}$. O ABS apresentou $\theta$ de $57,3^{\circ}$, classificado como uma característica hidrofílica $\left(\theta<90^{\circ}\right)$. Este valor é concordante com outros relatos da literatura para este material. ${ }^{41,42}$ Rocha e colaboradores ${ }^{42}$ avaliaram as características físicas do ABS e verificaram valor de $\theta$ em torno de $40,3^{\circ}$. O caráter hidrofílico do ABS pode ser determinante dependendo da aplicação do objeto impresso, sendo muitas vezes modificado para diminuir sua hidrofilicidade. O ABS apresenta propriedades mecânicas interessantes, com elevada resistência ao impacto e ductilidade. Contudo, possui menor resistência à tração e rigibilidade em comparação ao polímero PLA. ${ }^{43}$

O PLA apresentou $\theta$ de $66,2^{\circ}$, o que também foi similar a outros trabalhos, em que foram verificados valores variando entre $60^{\circ} \mathrm{e}$ $67^{\circ} \cdot{ }^{44-47}$ Assim, esse material também apresenta uma superfície com caráter hidrofílico. A capacidade de molhabilidade em água depende da natureza do material, sendo que o PLA é um poliéster alifático linear, polimerizado a partir de monômeros de ácido lático. ${ }^{48} \mathrm{O}$ material pode ser derivado da fermentação de amidos, sendo utilizadas matérias-primas renováveis (ou resíduos), tais como milho ou canade-açúcar. ${ }^{45}$ Devido a isso, obtém-se um polímero termoplástico com elevada biodegradabilidade e hidrofilicidade. ${ }^{46}$

Em comparação ao PLA, o ABS apresentou maior molhabilidade, o que pode estar relacionado à sua melhor capacidade de absorção de água. Segundo a literatura, isso pode ser atribuído à morfologia irregular formada após a extrusão dos filamentos e impressão em 3D. ${ }^{49}$ É importante destacar que ambas as peças foram impressas em 3D utilizando a técnica de FDM. A extrusão dos filamentos emprega temperaturas elevadas (ABS: $225{ }^{\circ} \mathrm{C}$, PLA: $180{ }^{\circ} \mathrm{C}$ ), o que pode ocasionar a formação de microfissuras na superfície do objeto impresso. Isso pode levar à penetração da água e ao aumento da molhabilidade das superfícies. ${ }^{50}$ Por este motivo, estas amostras podem ter apresentado um menor ângulo de contato, comparadas às demais superfícies analisadas.

A amostra de papel apresentou o maior valor de ângulo de contato $\left(82,8^{\circ}\right)$, representando caráter hidrofílico. ${ }^{40}$ É importante salientar que, independentemente do tipo, o papel é uma matriz extremamente complexa e heterogênea, tanto quimicamente, quanto morfologicamente. Assim, os materiais podem apresentar composição majoritária de compostos celulósicos (celulose, lignina e hemicelulose) e minoritária de minerais, tais como magnésio, silício, alumínio, fósforo, enxofre e cálcio. ${ }^{51,52}$ Além disso, alguns tipos de papel ainda podem conter aditivos ou resinas, o que leva à diminuição da molhabilidade e ao aumento do ângulo de contato.

O eletrodo de carbono vítreo (GCE) apresentou $\theta$ de $70,6^{\circ}$, valor considerado compatível com outros trabalhos da literatura. ${ }^{53,54}$ Por exemplo, Chen e colaboradores ${ }^{54}$ reportaram ângulo de contato de $76,5^{\circ}$ para a superfície do GCE. As características hidrofílicas podem ser interessantes do ponto de vista eletroquímico. Segundo os autores esta característica pode melhorar a biocompatibilidade da superfície eletródica, contribuindo para a imobilização de biomoléculas de maneira mais estável.

Outras superfícies analisadas foram eletrodos construídos com substratos considerados hidrofóbicos, sendo estes o papel impermeável (WP), o PVC e o PET. Para isso, os eletrodos foram fabricados empregando a técnica de silk screen, ou serigrafia, utilizando uma tinta condutora. O eletrodo em WP foi preparado com uma tinta composta por base de unhas e grafite, enquanto o eletrodo em PVC foi preparado com resina alquídica e grafite..$^{51} \mathrm{~A}$ partir dos resultados obtidos, constataram-se valores de ângulo de contato menores do que $90^{\circ}$, indicando que estas superfícies não apesentam caráter hidrofóbico. Isto pode sugerir que a presença das tintas condutoras pode ter alterado a capacidade de molhabilidade das superfícies. Neste sentido, Khakpour e colaboradores ${ }^{55}$ reportaram a diminuição do ângulo de contato de membranas de PVC após a modificação superficial com um compósito de óxido de grafenonanodiamante. Os autores relataram a diminuição de $\theta$ de $91,8^{\circ}$ para valores que variaram de $64,6-80,0^{\circ}$, dependendo da proporção de compósito adicionada. Esta alteração pode estar correlacionada à presença de grupos funcionais hidrofílicos no compósito, tais como $-\mathrm{OH}$ e $-\mathrm{COOH}$.

Diante do exposto, o desenvolvimento do dispositivo construído por impressão 3D permitiu a medição de ângulo de contato de diferentes superfícies, demonstrando a sua versatilidade e simplicidade de operação, além do baixo custo comparado ao equipamento comercial. Os resultados obtidos para este propósito, somado as vantagens da utilização por impressão em 3D, corroboram com a possibilidade do desenvolvimento de novos dispositivos ou equipamentos empregando esta estratégia, o que é extremamente atraente, principalmente do ponto de vista econômico.

\section{CONCLUSÃO}

A utilização da tecnologia de impressão 3D permitiu a criação de um novo dispositivo para medição de ângulo de contato, com relativo baixo custo e totalmente construído em laboratório. As peças foram impressas com elevada qualidade e precisão, o que permitiu a efetiva medição do ângulo de contato em diferentes substratos e 
Tabela 3. Medidas de ângulo de contato realizadas com o dispositivo 3D para diferentes materiais e superfícies eletródicas utilizando água deionizada

\begin{tabular}{lll}
\hline Superfície analizada & Medidas de ângulo de contato \\
\hline ABS & & \\
\hline & & \\
\hline
\end{tabular}

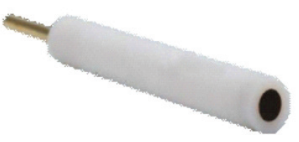

GCE
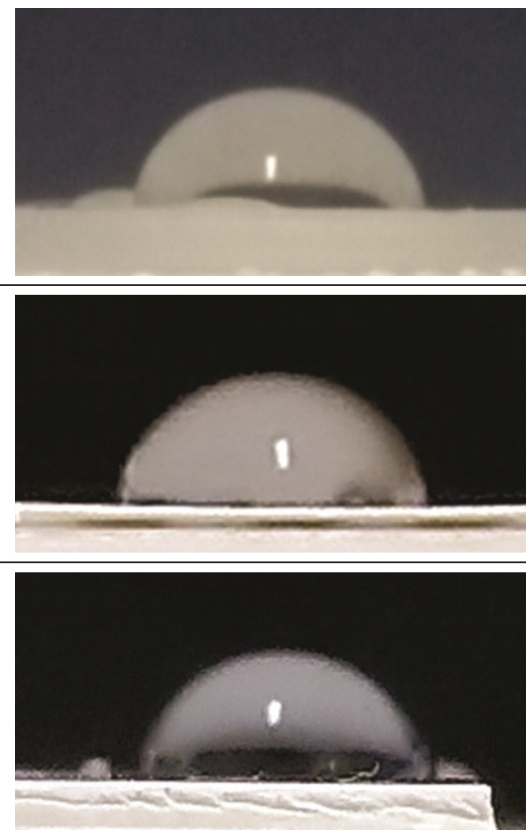

ABS: acrilonitrila-butadieno-estireno; PLA: ácido polilático; GCE: eletrodo de carbono vítreo; WPE: eletrodo impresso em papel impermeável; PVCE: eletrodo impresso em PVC.

eletrodos sólidos. A confecção do dispositivo também se mostrou de fácil operação em comparação a instrumentos comerciais, que normalmente necessitam de operadores com treinamento especializado. Isso demonstra a versatilidade da tecnologia $3 \mathrm{D}$, permitindo a confecção de dispositivos e equipamentos lab-made, viáveis e com uma ampla gama de aplicações. A presente proposta se mostrou bastante viável no que se refere a um dos principais objetivos, que é a utilização de uma ferramenta simples, versátil e de baixo custo na obtenção de um aparato ou suporte de medida. Isso permite que esta estratégia possa ser facilmente empregada no desenvolvimento de outros aparatos de maior ou menor complexidade, permitindo o avanço da pesquisa científica em grupos providos de baixo investimento financeiro.

\section{MATERIAL SUPLEMENTAR}

O tutorial de impressão 3D do dispositivo para medição de ângulo de contato e a tabela com o detalhamento das peças impressas para a confecção do dispositivo estão disponíveis em http://quimicanova. sbq.org.br, na forma de arquivo PDF, com acesso livre.

\section{AGRADECIMENTOS}

Esse trabalho foi realizado com fomento da: $i$ ) Coordenação de Aperfeiçoamento de Pessoal de Nível Superior (CAPES, Brasil) - Código de Financiamento 001; ii) Fundação de Amparo à Pesquisa do Estado de São Paulo (FAPESP) (processos 
2019/00473-2, 2019/01844-4, 2013/22127-2, 2017/21097-3 e 2017/23960-0).

\section{REFERÊNCIAS}

1. Lin, D.; Jin, S.; Zhang, F.; Wang, C.; Wang, Y.; Zhou, C.; Cheng, G. J.; Nanotechnology 2015, 26, 434003.

2. Weng, Z.; Wang, J.; Senthil, T.; Wu, L.; Mater. Des. 2016, 102, 276.

3. dos Santos, M. F.; Katic, V.; dos Santos, P. M. L.; Pires, B. M.; Formiga, A. L.; Bonacin, J. A.; Anal. Chem. 2019, 91, 10386.

4. Comina, G.; Suska, A.; Filippini, D.; Lab Chip 2014, 14, 2978.

5. Chen, X.; Liu, X.; Childs, P.; Brandon, N.; Wu, B.; Adv. Mater. Technol. 2017, 2, 1700148.

6. Mannoor, M. S.; Jiang, Z.; James, T.; Kong, Y. L.; Malatesta, K. A.; Soboyejo, W. O.; Verma, N.; Gracias, D. H.; McAlpine, M. C.; Nano Lett. 2013, 13, 2634.

7. Giannopoulos, A. A.; Mitsouras, D.; Yoo, S.-J.; Liu, P. P.; Chatzizisis, Y. S.; Rybicki, F. J.; Nat. Rev. Cardiol. 2016, 13, 701.

8. Kentzer, J.; Koch, B.; Thiim, M.; Jones, R. W.; Villumsen, E.; Resumos da $4^{\text {th }}$ International Conference on Mechatronics (ICOM), Kuala Lumpur, Malaysia, 2011.

9. Gonzalez-Gomez, J.; Valero-Gomez, A.; Prieto-Moreno, A.; Abderrahim, M. In Advances in autonomous mini robots, Springer: New York, 2012 p. 49.

10. Schelly, C.; Anzalone, G.; Wijnen, B.; Pearce, J. M.; Journal of Visual Languages and Computing 2015, 28, 226.

11. Petersen, E. E.; Kidd, R. W.; Pearce, J. M.; Technologies 2017, 5, 45

12. Pearce, J.; Organic Farming 2015, 1, 19.

13. Vaněčková, E.; Bouša, M.; Vivaldi, F.; Gál, M.; Rathouský, J.; Kolivoška, V.; Sebechlebská, T.; J. Electroanal. Chem. 2020, 857, 113760.

14. Ambrosi, A.; Webster, R. D.; Pumera, M.; Appl. Mater. Today 2020, 18, 100530.

15. Cardoso, R. M.; Kalinke, C.; Rocha, R. G.; dos Santos, P. L.; Rocha, D. P.; Oliveira, P. R.; Janegitz, B. C.; Bonacin, J. A.; Richter, E. M.; Munoz, R. A. A.; Anal. Chim. Acta 2020, 1118, 73, doi: 10.1016/j. aca.2020.03.028.

16. Skowyra, J.; Pietrzak, K.; Alhnan, M. A.; Eur. J. Pharm. Sci. 2015, 68, 11.

17. Andrade, D. C.; Ferraz, T. V. B.; Formiga, A. L. B.; Bonacin, J. A.; Quim. Nova 2020, doi: 10.21577/0100-4042.20170493.

18. Melnikova, R.; Ehrmann, A.; Finsterbusch, K.; IOP Conf. Ser.: Mater. Sci. Eng. 2014, 62, 12018.

19. Bak, D.; Assembly Automation 2003, 23, 340.

20. Rastogi, P.; Kandasubramanian, B.; Chem. Eng. 2019, 366, 264.

21. de León, A.; Domínguez-Calvo, A.; Molina, S.; Mater. Des. 2019, 182, 108044.

22. Baden, T.; Chagas, A. M.; Gage, G.; Marzullo, T.; Prieto-Godino, L. L.; Euler, T.; PLoS Biol. 2015, 13, e1002086.

23. Cardoso, R. M.; Castro, S. V.; Silva, M. N.; Lima, A. P.; Santana, M. H.; Nossol, E.; Silva, R. A.; Richter, E. M.; Paixão, T. R.; Munoz, R. A.; Sens. Actuators, B 2019, 292, 308.

24. dos Santos, P. L.; Katic, V.; Loureiro, H. C.; dos Santos, M. F.; dos Santos, D. P.; Formiga, A. L.; Bonacin, J. A.; Sens. Actuators, B 2019 $281,837$.

25. Kalinke, C.; Neumsteir, N. V.; Aparecido, G. O.; Ferraz, T. V. B.; dos Santos, P. L.; Janegitz, B. C.; Bonacin, J. A.; Analyst 2020, 145, 1207.
26. Leigh, S. J.; Bradley, R. J.; Purssell, C. P.; Billson, D. R.; Hutchins, D. A.; PloS One 2012, 7, e49365.

27. Foo, C. Y.; Lim, H. N.; Mahdi, M. A.; Wahid, M. H.; Huang, N. M.; Sci. Rep. 2018, 8, 1 .

28. Kalinke, C.; Wosgrau, V.; Oliveira, P. R.; Oliveira, G. A.; Martins, G.; Mangrich, A. S.; Bergamini, M. F.; Marcolino-Junior, L. H.; Talanta 2019, 200, 518 .

29. Li, F.; Macdonald, N. P.; Guijt, R. M.; Breadmore, M. C.; Anal. Chem. 2017, 89, 12805.

30. Ambrosi, A.; Pumera, M. J. A. F. M.; Adv. Funct. Mater. 2018, 28, 1700655.

31. Schneidereit, D.; Kraus, L.; Meier, J. C.; Friedrich, O.; Gilbert, D. F.; Biosens. Bioelectron. 2017, 92, 472.

32. Shaw, D. J.; Introdução a química dos colóides e de superfícies, Editora Blücher: São Paulo, 1975.

33. Leja, J.; Surface Chemistry of Froth Flotation; Springer US: New York, 2012.

34. Rabóczkay, T.; Fisico-quimica de Interfases, EDUSP: São Paulo, 2016.

35. Chau, T. T.; Bruckard, W. J.; Koh, P. T. L.; Nguyen, A. V.; Adv. Colloid Interface Sci. 2009, 150, 106.

36. Goh, G. D.; Yap, Y. L.; Tan, H.; Sing, S. L.; Goh, G. L.; Yeong, W. Y.; Crit. Rev. Solid State 2020, 45, 113

37. Stansbury, J. W.; Idacavage, M. J.; Dent. Mater. 2016, 32, 54.

38. Canessa, E. In Low-Cost 3D Printing for science, education and sustainable development; Canessa, E.; Fonda, C.; Zennaro, M., eds.; ICTP: Trieste, 2013.

39. Chen, H.; Muros-Cobos, J. L.; Amirfazli, A.; Rev. Sci. Instrum. 2018, $89,035117$.

40. Feng, L.; Li, S.; Li, Y.; Li, H.; Zhang, L.; Zhai, J.; Song, Y.; Liu, B.; Jiang, L.; Zhu, D.; Adv. Mater. 2002, 14, 1857.

41. Matheus, B. B.; Sandra, C. S. R.; Mater. Sci. Forum 2012, 727, 1616.

42. Rocha, S.; Donida, M.; Marques, A.; Can. J. Chem. Eng. 2009, 87, 695.

43. Rodríguez-Panes, A.; Claver, J.; Camacho, A. M.; Materials 2018, 11, 1333.

44. Alakrach, A.; Noriman, N.; Dahham, O. S.; Hamzah, R.; Alsaadi, M. A.; Shayfull, Z.; Idrus, S. S.; J. Phys. Conf. Ser. 2018, 1019, 012065.

45. Galindo, S.; Ureña-Núñez, F.; Rev. Mex. Fis. 2018, 64, 1.

46. Koo, G.-H.; Jang, J.; Fibers Polym. 2008, 9, 674.

47. Tham, C. Y.; Hamid, A.; Ain, Z.; Ahmad, Z.; Ismail, H.; Adv. Mater. Res. 2014, 970,324

48. Castillejos, S.; Cerna, J.; Meléndez, F.; Castro, M. E.; Aguilar, R.; Márquez-Beltrán, C.; González, M.; Polymers 2018, 10, 1184.

49. Lay, M.; Thajudin, N. L. N.; Hamid, Z. A. A.; Rusli, A.; Abdullah, M. K.; Shuib, R. K.; Composites, Part B 2019, 176, 107341.

50. Fernandes, J.; Deus, A. M.; Reis, L.; Vaz, M. F.; Leite, M.; Resumos da

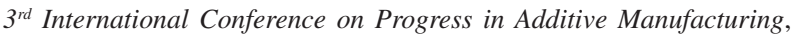
Singapore, Malaysia, 2018.

51. Camargo, J. R.; Andreotti, I. A.; Kalinke, C.; Henrique, J. M.; Bonacin, J. A.; Janegitz, B. C.; Talanta 2020, 208, 120458.

52. Fardim, P.; Holmbom, B.; Appl. Surf. Sci. 2005, 249, 393.

53. Shen, Q.; Wang, X.; J. Electroanal. Chem. 2009, 632, 149.

54. Chen, J.; Tang, J.; Yan, F.; Ju, H.; Biomaterials 2006, 27, 2313.

55. Khakpour, S.; Jafarzadeh, Y.; Yegani, R.; Chem. Eng. Res. Des. 2019, 152,60 . 